\title{
Stabilité el précision de la régulation dynamique
}

\section{Charles Coeurel}

Ingénieur à la Société du Canal de Provence

ef d'Aménagement de la Région Provençale

\section{Rappels fondamentraux sur le procédé de la régulation dynamique et méthode d'analyse}

La Régulation Dynamique est un procédé qui se distingue fondamentalement des régulations "classiques" sans même limiter ces dernières au domaine hydraulique.

On peut considérer que ce sont les quatre caractères suivants qui, dans leur ensemble, constituent l'originalité du procédé :

a) Il s'agit d'une régulation numérique programmée, de type discontinu et synchrone : c'est-à-dire que les réglages ne sont pas uniquement conditionnés par l'état du système à réguler mais qu'une "autorisation" extérieure au système délivrée par l'horloge interne du calculateur, est nécessaire pour que les commandes soient effectuées.

b) C'est une régulation de type prévisionnel, les prévisions intervenant à deux niveaux :

- d'abord pour la détermination des valeurs de référence des variables à réguler : les volumes de biefs (prévisions d'évolution des consommations),

- ensuite pour la correction des valeurs constatées de ces variables qui tient compte des écoulements transitoires en cours de propagation sur les biefs et résultant de réglages antérieurs.

c) C'est une régulation à caractère global, dans la mesure où chacun des organes de réglage n'agit pas seulement en fonction de l'état de la partie d'adduction ou "bief" qui lui est directement associée mais où, au contraire :

- chacun des biefs peut être appelé à participer à résorber une partie des écarts de volumes qui seraient apparus sur les biefs voisins,

- dans tous les cas, les besoins au niveau de chaque bief intègrent les modulations prévisionnelles des besoins des biefs aval. d) C'est une régulation à laquelle on peut imposer non seulement le respect d'un certain nombre de contraintes particulières d'exploitation, de type prédéterminé, mais également une régulation qui peut optimiser la conduite du fonctionnement avec comme objectif, par exemple, la réduction des frais de pompage ou la valorisation des turbinages.

Pour examiner les deux points de la stabilité et de la précision d'un procédé aussi complexe, on en schématisera le principe de fonctionnement.

A cet effet, la logique de la régulation sera décrite en utilisant les méthodes classiques d'analyse fonctionnelle des automatismes et en les appliquant au cas élémentaire de la régulation d'un bief considéré isolément.

Les conclusions de l'étude théorique seront étayées par l'analyse d'un essai effectué sur le bief aval du Canal de Provence.

Pour apprécier la précision du procédé dans un cas élémentaire mais concret, la valeur maximale de l'erreur cumulée, en volume, sera calculée en faisant abstraction des dispositions automatiques de "régulation globale" dont l'intérêt sera ensuite mis en évidence.

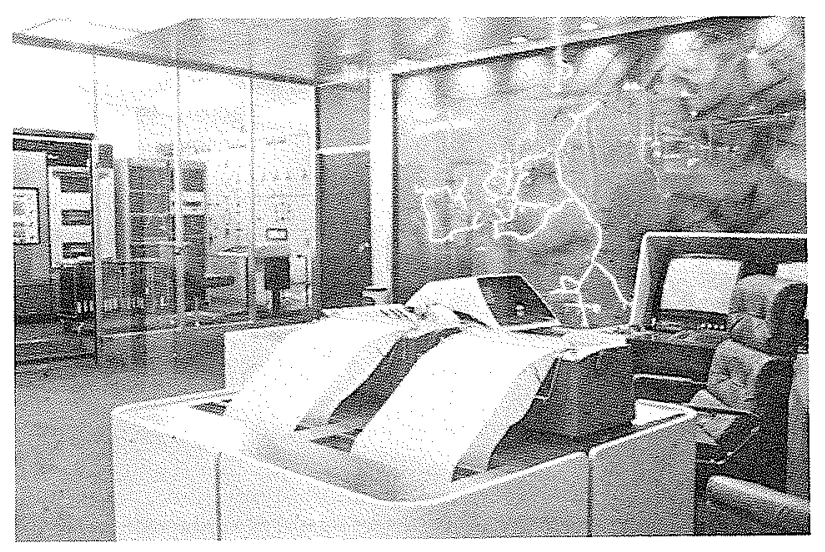

Le Centre général de Télécontrôle de la Société du Canal de Provence au Tholonet, près d'Aix-en-Provence 


\section{Schéma fonctionnel élémentaire de la régulation dynamique}

Considérons donc la partie de l'adduction comprise entre deux organes de réglage consécutifs, partie que nous conviendrons d'appeler "Bief".

Les opérations de réglage sont déclenchées par l'horloge interne du calculateur, en principe à intervalles de temps égaux : quand une bonne précision est nécessaire pour la régulation et dans le cas où les besoins sont susceptibles de présenter des modulations importantes, l'intervalle entre deux réglages consécutifs est le quart d'heure.

Chaque quart d'heure le calculateur doit donc déterminer l'ouverture de vanne, par exemple, qui va permettre de libérer au cours de la période de 15 minutes suivante un volume déterminé en tête du bief situé à l'aval immédiat de la vanne : soit $V d$ (volume délivré) cette valeur.

Pour cela, le volume d'eau en transit étant la variable à réguler, le programme déterminera :

- Vn, le volume nécessaire, c'est-à-dire la valeur de référence de la régulation à l'instant considéré. $V n$ est le volume qui correspond à la ligne d'eau qui devrait être celle de l'écoulement dans le bief pour satisfaire les besoins sur le cours du bief et à l'aval de celui-ci.

De façon pratique, cette ligne d'eau est obtenue en composant d'aval en amont les courbes des besoins prévisionnels relatives à chaque prise de l'adduction avec les déphasages de temps dus aux délais de propagation des ondes sur l'adduction.

- Ve, le volume effectif, valeur réelle du volume d'eau en transit dans le bief à l'instant considéré connue par l'intermédiaire des informations reçues en provenance du bief (niveaux, ouverture aval) corrigées le cas échéant des mouvements transitoires résultant des réglages antérieurs.

D'autre part, il faudra foumir en tête du bief pendant le quart d'heure à venir un volume $P$ correspondant aux prélèvements prévus à l'aval (supposons pour simplifier l'exposé que tous les prélèvements sont localisés à l'aval du bief) dans l'intervalle

$$
\left[H+D T, H+D T+15^{\prime}\right]
$$

où $H$ est l'heure du réglage et $D T$ le temps de propagation des ondes sur le bief. $[V d]_{H}$

On peut écrire qu'à l'heure $H$, le volume délivré sera

$$
[V d]_{H}=-k\left([V e]_{H}-[V n]_{H}\right)+[P]_{H}
$$

par rapport au réglage antérieur à l'heure $H O=H-15$

$$
[V d]_{H O}=-k\left([V e]_{H_{O}}-[V n]_{H O}\right)+[P]_{H O}
$$

On aura l'expression de la variation de réglage à effectuer

$$
r=[V d]_{H o}-[V d]_{H}=k(v-c)-p
$$

où $v=$ variation du volume réel du bief entre les 2 réglages successifs.

$c=$ variation du volume nécessaire $V n$ (variation de la consigne)

$p=$ variation de $P$

L'équation (1) peut être considérée comme l'équation de fonctionnement du "régulateur dynamique", avec les conventions de signes du schéma fonctionnel représenté sur la figure suivante :

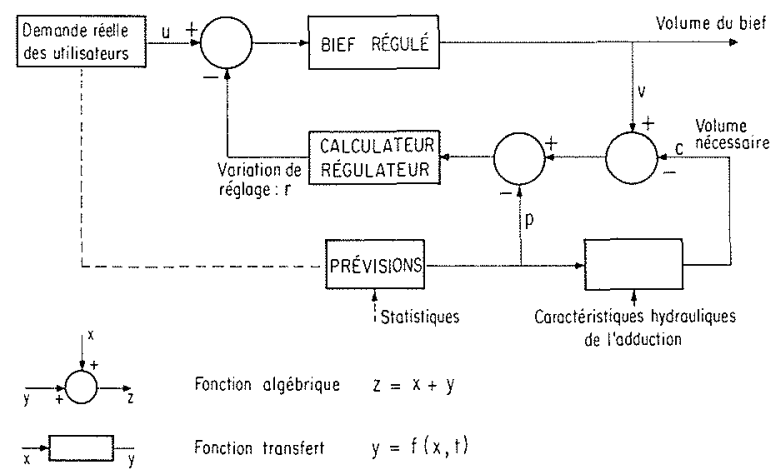

Le système régulé, c'est-à-dire le bief, peut être consiléré comme régi par une équation différentielle du premier ordre exprimée en fonction du volume :

$$
v+\tau \frac{d v}{d t}=u-r
$$

Dans ce système on peut admettre que l'élément "perturbateur" peut toujours se ramener à un écart $u$ entre demande réelle des utilisateurs $U$ et la prévision correspondante.

En combinant (1) et (2) il vient

$$
v+\tau \frac{d v}{d t}=u-k(v-c)+p
$$

Dans le cas général, les "prévisions" de besoins résultent de deux origines :

- Pour les prises "à la demande" des utilisateurs, elles sont automatiquement établies à partir des valeurs des consommations réelles $U$ enregistrées selon une expression du type

$$
P=a U^{b}
$$

où $a$ et $b$ sont des coefficients de corrélation établis statistiquement pour chacune des "phases" de la consommation journalière à l'intérieur desquelles l'évolution de cette consommation peut être assimilée à une droite.

- Pour les prises desservant des industries ou des communes dont la demande est en général régularisée par des réserves, les besoins peuvent ètre prédéterminés avec une assez bonne approximation suffisamment de temps à l'avance pour être directement entrées en mémoire du calculateur par l'exploitant.

On peut à ce niveau d'analyse du processus admettre que l'expression $p+k c$ de l'équation 3 peut se mettre sous la forme

$$
-\left(m_{1} \frac{d v}{d t}+m_{2}\right)
$$


la première partie de l'expression traduisant que la variation des facteurs prévisionnels qui interviennent dans la régulation sont dus, d'une part au gradient du volume du bief entrainé par l'écart sur les prévisions par rapport à la demande et d'autre part à l'erreur sur les besoins prédéterminés.

Finalement (3) s'écrirait sous la forme

$$
v(1+k)+\frac{d v}{d t}\left(\tau+m_{1}\right)=u-m_{2}
$$

La forme de cette équation correspond à une régulation proportionnelle et dérivée sur la résolution de laquelle nous ne nous étendrons pas sinon pour en rappeler sur le plan des résultats les 3 caractéristiques essentielles bien connues :

$\mathrm{Si}$, pour simplifier, on considère prévisions et demandes invariantes d'un quart d'heure à l'autre, - la valeur de l'écart volume du bief va, sous l'action de la régulation diminuer progressivement sans oscillation;

- cet écart va tendre vers un écart permanent, une fois le régime transitoire terminé $\left(\frac{d v}{d t}=0\right.$ dans l'équation

(4)), puisque $v$ ne devient pas nul mais tend vers $\frac{u}{1+k}$ dans l'hypothèse considérée où $m_{2}$ est nul et $u$ constant.

Il est évident que plus $u$, écart prévision-demande, est faible, plus l'écart résiduel est petit mais il est important de noter que l'écart se stabilise de toutes façons;

- enfin, la stabilisation est d'autant plus rapide que la "constante de temps" $\frac{\tau+m_{1}}{1+k}$ est faible c'est-àdire :

- que les variations de volume du bief sont peu sensibles aux écarts prévisions-demande ( $\tau$ faible) et aux modulations des prévisions ( $m_{1}$ faible),

- que les corrections du régulateur sont sensibles aux écarts de volume ( $k$ important).

\section{Résultats expérimentaux illustrant le caractère de régulation proportionnelle et dérivée de la régulation dynamique}

Après le développement théorique précédent, il est significatif de présenter les résultats d'essais effectués sur le Canal de Provence qui en constituent la vérification expérimentale.

L'essai a porté sur le bief aval de l'ouvrage qui est la cuvette de Signes-Malegorgue dont la longueur est de 1400 mètres et la pente 0,0003 . Cette cuvette est alimentée par le brise charge de Signes, sa capacité est de $10,5 \mathrm{~m}^{3} / \mathrm{s}$.

Lors de l'essai, la cuvette s'achevait par un batardage en amont de la galerie de Dubliou en cours de travaux; immédiatement avant ce batardeau, une vanne de fond permet de vidanger la cuvette vers le ruisseau du Latay à raison de $2,6 \mathrm{~m}^{3} / \mathrm{s}$ maxi de débit étalonné : c'est cet ouvrage qui a permis de simuler une prise.

L'essai décrit s'est déroulé en 3 phases le 21 avril 1976, il s'intégrait dans le cadre des essais dynamiques de mise en eau des ouvrages de la 2 ème tranche du Canal de Provence.

Phase 1 : Programmation dès 7 heures par l'exploitant sur le calculateur d'un besoin de $26001 / \mathrm{s}$ sur la prise de la vidange de Malegorgue pour 11 heures 30 .

Phase 2 : Non ouverture de la vidange à 11 heures 30 et maintien du débit prélevé nul pendant 3 heures sans modification des besoins programmés.

Phase 3 : Ouverture de la vidange à 14 heures 30 seulement, c'est-à-dire avec 3 heures de retard.
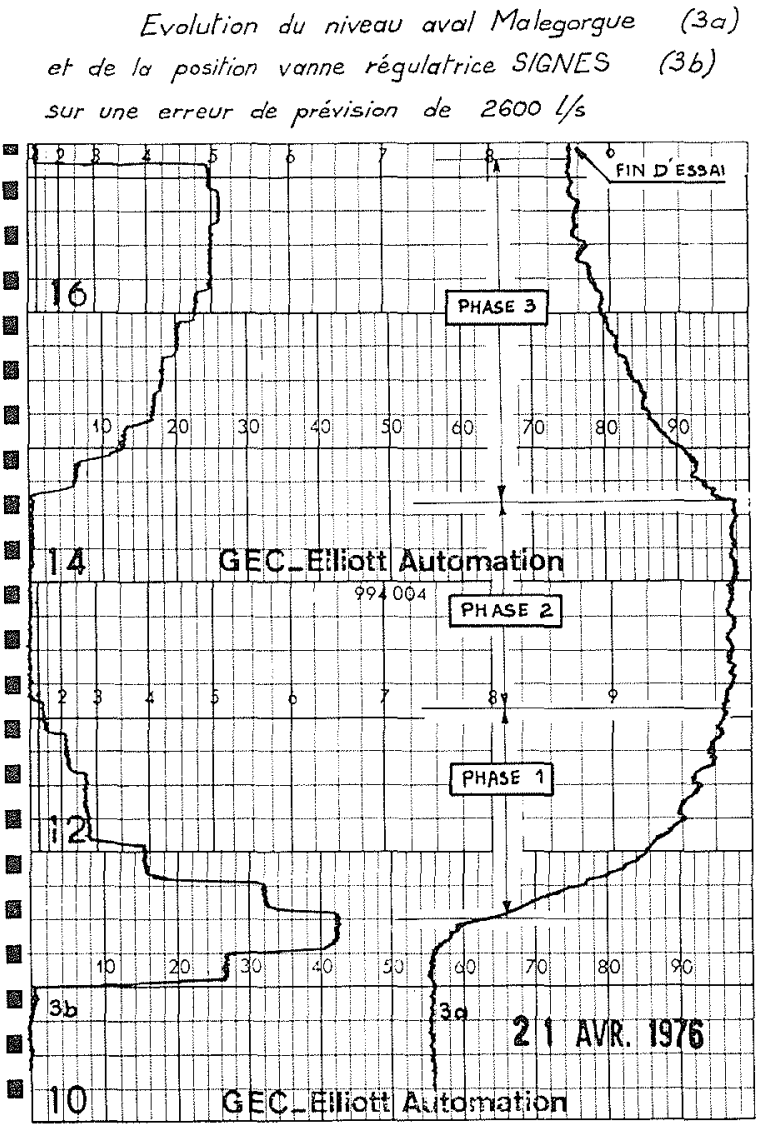

L'enregistrement ci-dessus retrace pendant ces 3 phases l'évolution dans le temps :

- du niveau à l'aval de la cuvette à partir d'une valeur correspondant à l'état initial de la cuvette (courbe 3a)

- du pourcentage d'ouverture de la vanne régulatrice du brise charge de Signes directement commandée par le calculateur (courbe 3b).

L'échelle des temps, en vertical, est graduée en quarts d'heure. En horizontal, les 2 mesures sont exprimées en pourcentage de la course totale des capteurs (pour le niveau, $100 \%$ correspondant à 2 mètres, soit 5 centimètres au-dessous du niveau du déversoir de la cuvette à berges inclinées parallèlement au radier).

On constate, au cours de la première phase,l'ouverture $\mathrm{du}$ brise charge anticipant sur les besoins programmés de façon à satisfaire la demande prévue pour 11 heures 30 . 
Du fait de la non ouverture de la vidange à 11 heures 30, "l'erreur de prévision" se traduit par une montée du plan d'eau anormale.

Dès l'amorce du dépassement de la cote correspondant au "volume nécessaire", le brise charge cherche à corriger l'erreur et réduit progressivement le débit jusqu'à se refermer totalement, bien que l'erreur de prévision soit maintenue.

Lorsqu'à 14 heures 30 , l'ouverture de la vidange est réalisée, le niveau se stabilise à la valeur correspondant à l'écoulement à $2,6 \mathrm{~m}^{3} / \mathrm{s}$ compte-tenu en l'occurence d'une contrainte qui avait été imposée pour l'essai et entrainait le passage de ce débit sous un niveau aval correspondant à l'écoulement du débit normal de $10,5 \mathrm{~m}^{3} / \mathrm{s}$ pour rendre le résultat plus significatif.

On constate qu'entre cette dernière valeur et la surélévation maximale, la différence de niveau correspond au passage de $76 \%$ à environ $97 \%$ soit 42 centimètres pris sur la revanche normale de la cuvette sans qu'un déversement ne se soit produit.

Sans aller plus avant dans l'analyse de l'essai, on considèrera que la situation du bief en extrémité aval d'une adduction jointe aux conditions particulières de l'essai :

- débit sur le bief limité à l'alimentation de la prise,

- erreur de 100 pour 100 sur les prévisions,

- non mise en jeu de l'aspect global de la régulation dynamique pour écrêter l'erreur (voir plus loin le chapitre précision),

permettent d'illustrer le cas de fonctionnement schématique de la régulation d'un bief isolé décrit au paragraphe précédent.

\section{Stabilité de la régulation}

Des caractéristiques fonctionnelles de la régulation dynamique on déduira que les risques d'instabilité du processus sont pratiquement inexistants pour une série de raisons liées à la nature et aux modalités de cette régulation.

En particulier :

- Le caractère proportionnel et dérivé de la régulation élémentaire n'est tout d'abord de nature, ni à entretenir, ni à fortiori à créer une instabilité de la variable régulée : ceci en raison de l'allure non oscillante de la courbe de retour à l'équilibre du volume, parfaitement mise en évidence pour l'essai effectué.

- Le principe même de la connaissance par le système des valeurs des volumes de bief rend difficile la fluctuation non significative de ces valeurs susceptible d'instaurer des conditions d'instabilité du processus, d'origine extérieure : les volumes sont en effet des variables calculées à partir d'informations élémentaires de natures différentes (niveaux, positions de vannes) préalablement filtrées physiquement dans l'installation du capteur mais aussi mathématiquement par programme, à la réception des mesures.
- Le caractère discontinu de la régulation dynamique et plus précisement son caractère synchrone font que le régulateur est, dans une certaine mesure, "découplé" du système à réguler. A un état de bief ne correspond pas nécessairement un réglage car le réglage pour être effectué doit être "autorisé" par une origine extérieure au processus, en l'occurence l'horloge interne du calculateur. Entre 2 réglages au cours desquels le calculateur règle des ouvertures de vannes et non des débits de consigne, le système ne peut qu'être stable.

- Le caractère global de la régulation permet d'écrêter les écarts de volume dans les biefs lorsque ceux-ci dépassent certains seuils, on le verra dans la suite : dans l'éventualité où une instabilité aurait pris naissance dans un bief, la régulation globale en écrêterait l'amplitude et la désamorcerait.

Le principe de la régulation, la confirmation obtenue non seulement par les essais effectués mais par plusieurs années d'exploitation du système dans des circonstances nécessairement diverses permettent de garantir à priori la stabilité de la régulation dynamique, sans imposer de contraintes d'exploitation pour limiter par exemple les variations de débit rapides et importantes sur les adductions.

A cet égard, il est à noter qu'une des difficultés majeures d'exploiter des adductions mixtes équipées sur certains tronçons de régulations conventionnelles ayant pourtant fait l'objet d'études de stabilité, est que la régulation de ces biefs n'est pas compatible avec les variations de régime que tolèrent parfaitement les tronçons en régulation dynamique.

\section{La précision de la régulation dynamique}

On a montré comment la régulation dynamique dans des conditions d'essai très particulières laissait subsister un écart résiduel qui, dans l'exemple considéré, pouvait toutefois être absorbé par la revanche normale du canal.

L'expérience réalisée est défavorable à deux points de vue pour juger de la précision réelle de la régulation :

a) elle supposait une erreur de prévision de 100 pour 100 sur les besoins,

b) elle faisait abstraction des possibilités de gestion globale de la régulation dynamique.

Noter que, sur le premier point, le cas d'une erreur de cet ordre ne peut être exclu sur incidents pour certains types de prises : par exemple dans le cas de stations de pompage dont le fonctionnement viendrait à s'interrompre brutalement à la suite d'un arrêt de l'alimentation électrique.

Dans le cas général, cependant, la précision des prévisions et leur réactualisation lors de chaque cycle de réglage font que l'erreur résiduelle est très limitée.

Pour en chiffrer la valeur maximale, le raisonnement suivant permet de simuler valablement les modalités de la mise en jeu des prévisions dans le cadre de la régulation. 
Considérons un bief desservant à son extrémité aval la prise d'un réseau de distribution fonctionnant à la demande des utilisateurs.

La demande journalière en période de pointe est représentée par la courbe type (1) qui servira de base de raisonnement.

Les modalités du programme de prévision conduisent, à partir du volume $U$ enregistré au cours du dernier quart d'heure écoulé, à déduire de proche en proche les consommations $P$ des périodes successives à venir par des équations de la forme

$$
P=a U^{b}
$$

où $a$ et $b$ sont des coefficients établis statistiquement pour chacune des périodes de la journée et pour chacune des prises.

L'expérience a montré que l'erreur relative $E$ maximale sur les volumes $P$ ainsi prévus peut être représentée par la droite

$$
E=1,5 N
$$

où $E$ est l'erreur relative exprimée en pourcentage, et $N$ le nombre de demi-heures d'anticipation de la prévision.

L'erreur maximale ainsi définie couvre plus de 95 pour cent des cas statistiquement étudiés dans des conditions d'exploitation qui incluent des cas de variation exceptionnelle de la demande dus par exemple à l'arrivée brutale d'orages en période de pointe d'irrigation.

Cette loi traduit évidemment le fait que l'erreur relative possible est d'autant plus importante que la prévision porte sur un délai plus long et donc que la longueur du bief est plus grande.

Supposons, pour simplifier, que les cycles de réglage coïncident avec les cycles de révision des prévisions et que la durée entre les cycles soit d'une demi-heure, temps qui définira la période élémentaire qui va servir de base aux calculs suivants.

Soit $N$ le nombre de périodes élémentaires, donc de demi-heures, sur lequel, compte tenu du temps de réponse du bief, doit porter la prévision lors de chaque réglage.

En partant d'un état d'équilibre supposé du bief (volume effectif $=$ volume nécessaire) le premier réglage considéré va être effectué en introduisant pendant la demi-heure au cours de laquelle il va se maintenir une erreur maximale

$$
E_{N} V_{l}
$$

où $E_{N}$ : erreur relative pour une prévision portant $\operatorname{sur} N$ demi-heures

et $V_{1}$ : volume prévu pour la consommation de la prise aval lors de la première demi-heure postérieure de $N$ demi-heures au réglage initial.

Lors du réglage suivant, à l'erreur précédente va s'ajouter sur le bilan une erreur relative à $V_{2}$ :

$$
E_{N} V_{2}
$$

mais le réglage effectué visera en outre à corriger une partie de l'écart du précédent réglage puisque, alors, la prévision concernant la consommation $V_{1}$ peut être

\section{Courbe 1}

Evolution type de la demande journalière des réseaux en période de pointe d'irrigation
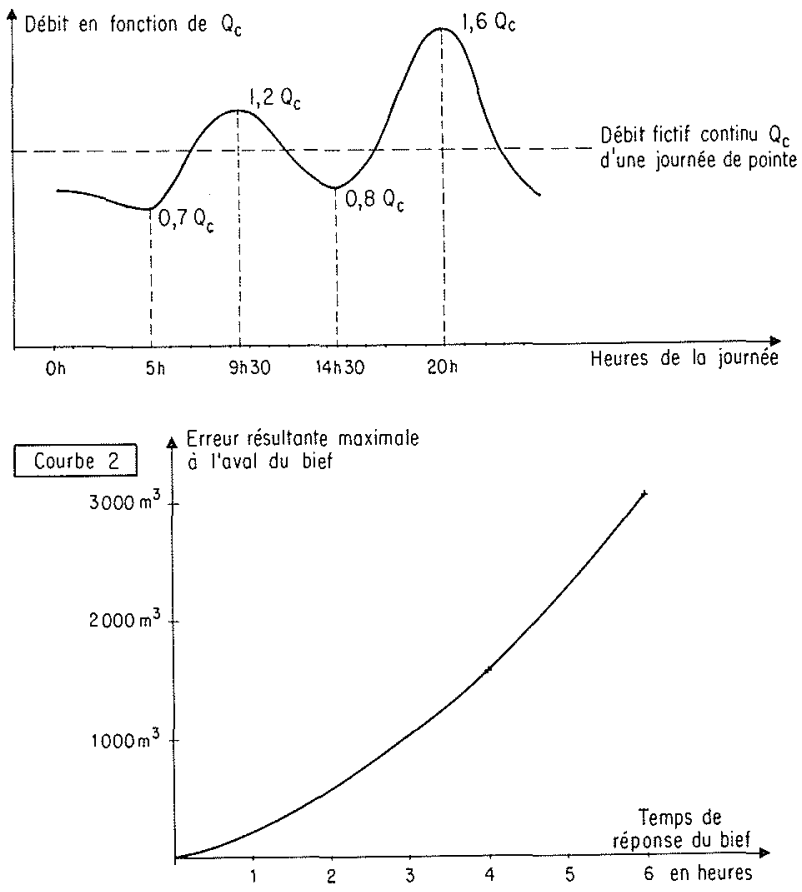

Lo courbe ci-dessus correspond a un bief desservant une prise donf le débit fictif $Q_{c}=1 \mathrm{~m}^{3} / \mathrm{s}$

appréciée plus précisément. L'erreur précédente va donc être corrigée partiellement de la valeur :

$$
E_{N} V_{1}-E_{N-1} V_{1}
$$

Ainsi de suite ... on arrive facilement à l'expression de l'erreur maximale résultante qui se cumulerait à l'aval du bief

$$
V=E_{1} V_{1}+E_{2} V_{2}+E_{3} V_{3}+\ldots+E_{N} V_{N}
$$

Tous calculs faits à partir des éléments chiffrés extraits de la courbe de la demande journalière et de la droite permettant de déterminer la valeur des erreurs relatives, les valeurs de $V$ sont données par la courbe 2 calculée en fonction des temps de réponse du bief en considérant les périodes les plus défavorables de la journée, c'est-à-dire celles mettant en jeu les plus forts volumes consommés aux alentours de la pointe de consommation du soir.

Sur cette courbe, $V$ est exprimé en mètres cubes pour une prise dont le débit fictif continu journalier est de 1 mètre cube par seconde.

Le résultat montre que l'écart maximum cumulé sur le volume du bief entraîné par l'imprécision de la régulation liée aux prévisions est relativement faible. Cet écart peut dans la plupart des cas être absorbé par les revanches habituelles sur un canal à berges parallèles au radier.

Le processus de régulation globale écrêterait en tout état de cause, si nécessaire, les erreurs supérieures qui amèneraient les niveaux à dépasser certaines limites, supérieures ou inférieures, prédéterminées. Ce processus est dans la pratique mis en jeu exceptionnellement à la suite d'incidents sur certaines télécommandes ou 
pour des variations accidentelles et brutales du débit prélevé par des prises comme des stations de pompage.

Les modalités d'application des dispositions d'interaction des biefs, voire d'utilisation des capacités dispo- nibles sur certains réservoirs dont la gestion est intégrée à celle de l'adduction, font l'objet de programmes spécifiques adaptés à la configuration du réseau régulé et aux disponibilités utilisables sur chacun des biefs ou des réservoirs au moment de l'incident.

\section{Discussion}

\section{Président: M.M. LECRIQUE}

M. le Président remercie M. COEURET pour son exposé fort documenté et ouvre la discussion.

Un régulateur de type proportionnel et intégral, remarque M. GAUTHIER (SOGREAH) n'est pas forcément "oscillant" comme l'exposé peut le laisser penser ; c'est un problème de choix des paramètres du système. D'autre part :

- Quelle est la période de commande de la régulation ; estelle uniforme pour tout le réseau?

- La variable de règlage choisie est le volume d'eau existant dans le bief ; n'y-a-t-il pas des cas où la variable à prendre en compte est un niveau?

M. COEURET est bien d'accord sur le fait que le choix des paramètres peut amortir le caractère oscillant d'une régulation dû à l'action intégrale et rappelle qu'une régulation peut être oscillante sans être instable, c'est ce qu'a probablement voulu dire M. GAUTHIER .

En ce qui concerne la période de commande, il précise : $\mathrm{Au}$ début, nous avions des cycles de réglage toutes les demi-heures. Puis nous avons été amenés sur les nouveaux ouvrages du Canal de Provence à réduire la périodicité au quart d'heure essentiellement pour des raisons de sécurité.

Compte tenu des débits transitant actuellement dans le canal, des corrections de réglage tous les quarts d'heure sont peut être un luxe mais elles nous garantissent contre d'éventuels incidents quand des variations brutales et importantes du débit appelé peuvent être ređoutées ; par exemple lorsqu'un bief alimente une station de pompage qui risque de s'arrêter intempestivement de fonctionner à la suite d'une disjonction (cas de la partie aval de la branche de Bimont). Dans ce cas, en effet une périodicité trop longue entre deux règlages peut entraîner une accumulation importante de volume avant que la correction nécessaire ne s'effectue.

Nous avons été amenés en particulier pour des études de modernisation de canaux anciens dans la Basse Durance à prévoir des cycles de réglage beaucoup plus espacés, répond M. COEURET

S'agissant de canaux anciens, sur des longueurs importantes (de l'ordre de $50 \mathrm{~km}$ ), on n'avait prévu que 4 ou 5 capteurs de niveau. En conséquence, compte tenu du temps de réponse hydraulique du canal, il s'écoulerait, grosso modo, une heure avant qu'un règlage en tête du canal se soit matérialisé par une variation de niveau au droit des premiers capteurs implantés le long de l'ouvrage. Il était donc inutile de prévoir des cycles de réglage plus rapprochés puisqu'on n'avait pas la possibilité d'apprécier l'incidence d'un règlage antérieur.

Revenant à la deuxième question posée par M. GAUTHIER, M. COEURET déclare :

En effet, ce sont "les volumes" qui sont régulés mais ceuxci se déduisent des mesures de niveau reçues. En simplifiant, le calculateur part du niveau et du débit à l'aval du bief à l'instant considéré et prend en compte les phénomènes en cours de propagation sur la partie amont du bief consécutif aux réglages antérieurs ; finalement on suit de façon très stricte l'évolution des niveaux. Dans les derniers ouvrages mis en service, il existe 3 ou 4 capteurs de niveau dans les 500 derniers mètres des biefs; nous avions pensé qu'il était particulièrement important de bien connaître la ligne d'eau dans cette partie du remous. En fait, cette multiplication des capteurs de niveau nous permet surtout d'éliminer les informations aberrantes et de repérer le dérèglage d'un capteur ; cela est important sur le plan de la sécurité.

Pour calculer le volume effectif existant dans le bief, à partir des niveaux relevés, quelles formules utilisez-vous? demande M. SBIHI.

Par des calculs préalables systématiques, explique M. COEURET, nous déterminons pour chacun des biefs les lignes d'eau en régime permanent (c'est-à-dire stabilisé) pour une série de débits et des conditions aval déterminées ; il s'agit d'un calcul classique de courbe de remous. Nous en déduisons des tables à double entrée qui donnent le volume existant dans le bief pour des conditions de débit et de niveau à l'aval données. Ce sont ces tables qui sont exploitées en temps réel par le calculateur industriel ; le caractère non-permanent de l'écoulement est pris en compte par une correction faite par l'ordinateur d'après la mémorisation des réglages antérieurs en cours de propagation.

Que se passe-t-il en cas de panne des équipements de télétransmission ou des ordinateurs de commande ? interroge M. BRISSAUD (SPIE-Batignolles).

Au niveau des supports de télétransmission, dit M. COEURET, nous avons réalisé un réseau maillé qui nous permet, a priori, de nous garantir contre les coupures de cables.

Le système de télécontrôle comporte un certain nombre de stations qui regroupent les informations ; sur les derniers réseaux mis en service, nous avons doublé ces stations. Lorsque l'une de celles-ci est en panne, nous avons ainsi une station de secours prête à la suppléer. Nous avons même développé un système assez sophistiqué mais délicat à mettre au point pour que les basculements sur les équipements de secours s'effectuent automatiquement en cas de défaut.

En outre, la régulation dynamique étant une régulation globale, quand bien même le calculateur manquerait d'informations sur une partie du canal, le programme essaie - par les autres informations dont on dispose à l'amont et à l'aval et par la mémorisation des réglages antérieurs - de réaliser un réglage - évidemment un peu moins précis que lorsque tout le système marche suffisamment correct pendant le temps d'intervention et de réparation. L'inertie du système hydraulique est suffisante pour qu'en général une intervention de quelques heures, à la suite d'un incident sur le système de régulation, ne provoque pas de dérèglement dangereux.

M. le Professeur THIR RIOT apporte l'information ci-après : Il y a une douzaine d'années, j'avais à la demande d'E.D.F., étudié les courbes de remous "à volume constant" dans le canal d'amenée à surface libre d'une usine hydroélectrique. Je m'étais rendu compte que toutes les lignes d'eau passaient au voisinage d'un même point du canal, de sorte que l'on pouvait considérer, en première approximation, que les plans d'eau oscillaient autour d'un axe horizontal, placé en ce point.

Avez-vous rencontré ce phénomène au cours des nombreux calculs de courbes de remous que vous avez effectués? demande M. THIRRIOT.

La régulation dynamique n'est pas une régulation à volume constant rappelle $M$. COEURET mais il serait fort intéressant de rechercher dans les nombreux calculs et essais effectués sur le Canal de Provence les constatations faites par M. le Professeur THIRRIOT. 


\section{Abstract \\ Stability and accuracy of a «dynamic control» system}

\section{Main features of a dynamic control system}

a) Intermittent programmed control synchronized by the computer timer.

b) The control system "anticipates" demand variation and other factors.

c) Overall control of supply canal or duct sections and branch reservoirs.

d) Optimization of canal duct flow conditions where necessary to reduce pumping cost or ensure maximal economic efficiency of run-of-the river turbine operation.

\section{Basic operating principle}

Consider a single canal section (a "reach" between two control works) :

The system controls the volume of water in the reach at regular intervals, e.g. every 15 minutes, so that it delivers a given volume of water (Vd) for the following purposes:

a) To correct the difference between the effective volume in the reach (Ve) (known from measurement data) and the required volume ( $\mathrm{Vn}$ ) determined from the computer's demand forecast for the time taken by the waves to travel the length of the reach $(\Delta \mathrm{T})$.

b) To meet the demand during the following period:

$$
[H+\Delta T] \text { to }\left[H+\Delta T+15^{\prime}\right]
$$

Where $\mathrm{H}$ is the hour at which control is effected.

The corresponding operating equation is determined by automatic system analysis methods. It expresses the proportional differential action of elementary dynamic control.

\section{Experimental verification}

A test with a simulated forecasting error of $100 \%$ on the downstream reach of the Canal de Provence confirmed that the control error stabilised without oscillation at a value corresponding to the "residual error" of the control system and depending on the degree of inaccuracy of the forecast.

Control stability

Considering its method of application, dynamic control can be considered stable, since action of the control device is sufficiently "uncoupled" from the system under control.

\section{Control accuracy}

For offtakes operating "on demand", the forecasting principle is based on extrapolation of consumption data recorded when control takes place, using correlation laws with stasticallydetermined parameters.

The maximum possible error downstream from the considered reach is estimated from the following:

a) Relative forecasting error $E$ for the time (in half-hours $N$ ) covered by the forecast.

b) The representative daily peak consumption curve for an offtake supplying a mean daily discharge of $1 \mathrm{~m}^{3} / \mathrm{sec}$.

c) Reaction time of the considered reach, i.e. its length.

The calculated data plot shows this error to be sufficiently small to be usually offset by normal canal freeboard, without any requirement for raised horizontal canal banks (i.e. civil engineering costs are lower than for conventional forms of control).

Should this error unexpectedly exceed a given critical value, the general control arrangements for all canal sections discharge part of the surplus to reaches farther downstream or divert it to branch storage facilities, or they initiate other forms of appropriate action. Similar automatic action is also initiated in the event of a probable shortage, e.g. drawing of supplies from "over-stocked" reaches or spreading the shortage out over several reaches to make it barely noticeable for consumers during the time required for supply corrections to react along the system. 